\title{
Enter the era of facilitated anastomotic devices for coronary bypass surgery
}

Hani Shennib, MD

From the Department of Cardiothoracic Surgery, McGill University Health Center, Montreal, Quebec, Canada.

Received for publication Dec 19, 2000; accepted for publication Dec 28, 2000.

Address for reprints: Hani Shennib, MD, 1650 Cedar Ave, Room L9-121, Montreal, Quebec H3G 1A4, Canada.

J Thorac Cardiovasc Surg 2003;125:S78-9 Originally published in J Thorac Cardiovasc Surg 2001;121:833-4.

Copyright $\odot 2003$ by The American Association for Thoracic Surgery

$0022-5223 / 2003 \$ 30.00+0$

doi: $10.1067 / \mathrm{mtc} .2003 .234$

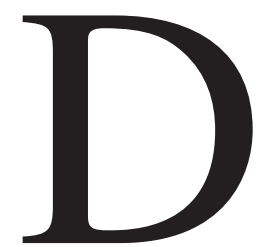

uring the past 50 years and except for some attempts to use mechanical staplers by the Russian pioneer surgeon Kolosov, almost all proximal and distal aorta-coronary anastomoses have been done with conventional hand-held sutures. The comfort to surgeons in performing a reliable anastomosis with conventional suturing has led to its adoption as the gold standard and will likely be the principal reason for hesitancy in adopting alternate anastomotic methods. So why change?

Unquestionably, the principal impetus for change in our cardiac surgical practice has been the desire to offer patients less-invasive therapies, particularly in light of the rapid growth of percutaneous catheter-based interventional cardiology procedures. Since 1995 and with growing interest in developing platform technology for minimally invasive coronary artery bypass grafting (CABG), numerous concepts, devices, techniques, and technologies have been introduced. Some have been adopted. Others have vanished despite exhaustive efforts by their industrial promoters. (Surgeons may become excited quickly by new devices, but they also have the intellectual and practical honesty to abandon those that are of no benefit to their patients.) In fact, in a review of all the instruments developed-including cameras, scopes, retractors, occluders, and others-stabilizers for coronary artery anastomosis stand alone as the most significant contributors to the growth of beating-heart CABG. Of all the innovative technology to be considered in the near future, anastomotic devices, in my opinion, will have the greatest impact on the practice of CABG.

In general, for any anastomotic device to become widely accepted, it must fulfill three essential characteristics: facility, precision, and evidence of long-term effectiveness. A device that is cumbersome to set and deploy during surgery will not likely survive early validation. It must be easy to use.

One of the key advantages of hand-held suturing devices is the ability to tailor each bite to the exact characteristics of the graft and native vessel. An anastomotic device must be appropriate for the diseased vessels and for the exact angulation and layout of the surgical field. Edges must be well coapted with proper sealing and no impediment to flow. Most important, however, as in all revascularization innovations, the device must provide satisfactory long-term patency. Recent interest in performing $\mathrm{CABG}$ through keyhole incisions has intensified efforts to resolve the problem of how to perform coronary artery anastomoses through limited access using endoscopic principles. Endoscopic microsuturing is extremely difficult. Indirect visualization via a camera that replaces direct vision, coupled with the use of rigid, long instruments, renders the results of endoscopic suturing less than desirable. Ironically, very sophisticated and expensive robotic technology has been suggested to facilitate performance of endoscopic anastomosis, but in a conventional suturing manner, ${ }^{1}$ rather than through alternative anastomotic concepts. It is for this reason that robotic technology has failed so far to simplify CABG.

Automated and semiautomated suturing devices are now on the horizon. ${ }^{2}$ Also promising is technology that shies away completely from traditional suturing. At least eight medical technology companies are presently focused on developing anastomotic devices to replace conventional suturing for proximal and distal CABGs. 
Challenges confronting this "alternative anastomotic technology" include success in three areas: delivery, deployment, and durability.

In validating this new technology, surgeons have an advantage over interventional cardiologists in the ability to access the chest cavity directly. This will likely expedite the testing of some anastomotic devices, initially through open chest methods and without the limitations of complex designs and material made to suit endovascular access. Catheter-based endovascular delivery, which should be the ultimate objective, will necessitate the integration of imaging technology into the operating rooms and the training of surgeons on catheter-based techniques. Deployment of new anastomotic devices in more challenging and complex native aortas and diseased coronary arteries will likely be the Achilles' heel of such devices for some time. Concern with biocompatibility, thrombosis, and intimal proliferative response will necessitate long-term pre-marketing studies for many of the devices.

In this issue of the Journal, Calafiore and colleagues ${ }^{3}$ present cutting-edge experience with a new anastomotic device for performance of proximal CABGs. The principle of the device is the deployment of a metal connector mounted within a vascular conduit onto the wall of the aorta. Like most coronary stents, the device is made of inert metal with memory. It anchors the graft to the wall of the aorta by recoiling to its original design. Clinical experience from other companies with different designs will be forthcoming. Nitinol stents are only one category of devices that will soon become available and commonplace in cardiac surgical practice. Other couplers, molds, and stents based on different biocompatible material are currently being developed. Calafiore and associates have shown that it is possible to implant one such anastomotic device with reasonable facility and safety. Before stocking our operating room shelves with it, however, we will need more scientific validation on the safety, the efficacy, and certainly the long-term angiographic follow-up of anastomoses created with this device to confirm its durability.

We also do not yet know whether these anastomotic devices are practical in aortas with normal wall thicknesses only or for diseased aortic tissue of varying thicknesses and densities. Furthermore, is this technique applicable to vein grafts of average diameter only, or could it be used for smaller and larger veins? What about arterial grafts? Will they sustain the shear force of mounting and deploying the anastomotic device?
So what potential benefits to patients will this technology offer?

First, this device allows the performance of a quality anastomosis repeatedly, with consistency, and with minimal intersurgeon variation. For the first time ever, the coronary anastomosis will be determined not by the skill of the surgeon but by the effectiveness of the device. Second, the device is likely to expedite the procedure and allow several proximal anastomoses to be done in very little time. Third, it represents yet another facilitator for performing more off-pump beating-heart CABGs. Perhaps the most significant potential advantage of using this and other forthcoming proximal aorta-coronary anastomotic devices is the likelihood of less manipulation of the aorta, which will decrease the risk of aortic embolic showering to the brain and other organs.

What are the drawbacks?

The only one that I could think of, provided that this technology will ultimately be applicable to all types and sizes of vascular grafts, is the high cost. Assuming $\$ 330$ for a single application, another $\$ 1000$ will be added to a triple bypass operation. This is an untenable price for CABG, a procedure already limited by other economic restrains. However, by using this and other facilitating technology, it may be possible to package a new less-invasive CABG procedure with reduced operative and postoperative time and a lower risk of stroke. As more devices are manufactured and used, the price will be reduced.

CABG surgery will never be the same. The drive toward new less-invasive strategies will assure not only continuous improvement of surgical outcomes but also an appreciation of the desire for patients to undergo less onerous operations, competitive with current percutaneous interventional cardiology procedures. As long-term results of coronary stents continue to improve, we as surgeons must respond by advocating new and potentially less-invasive procedures that offer the only remaining advantage for continuing CABG today: long-term efficacy.

\section{References}

1. Shennib H, Bastawisy A, Mohr F. Robotic assisted telemanipulation enhances endoscopic coronary artery bypass. J Thorac Cardiovasc Surg. 1999;117:310-3.

2. Shennib H, Korkola S, Bousette N, Giaid A. An automated interrupted suturing device for coronary artery bypass grafting: automated coronary anastomosis. Ann Thorac Surg. 2000;70:1046-8.

3. Calafiore AM, Bar-El Y, Vitolla G, Di Giammarco G, Teodori G, Iacò AL, et al. Early clinical experience with a new sutureless anastomotic device for proximal anastomosis of the saphenous vein to the aorta. J Thorac Cardiovasc Surg. 2001;121:854-8. 